\title{
Psychoacoustic Characteristics of Tinnitus versus Temporal Resolution in Subjects with Normal Hearing Sensitivity
}

\author{
Ola Abdallah Ibraheem ${ }^{1}$ Mohammad Ramadan Hassaan ${ }^{1}$ \\ ${ }^{1}$ Audiology Unit, Department of Otolaryngology, Zagazig University, \\ Address for correspondence Ola Abdallah Ibraheem, 40-B Abdul Aziz \\ Zagazig, Egypt \\ Ali street, Zagazig, Sharkia, Egypt (e-mail: ola_medvoice@yahoo.com).
}

Int Arch Otorhinolaryngol 2017;21:144-150.

\begin{abstract}
Introduction Cochlear or neural mechanisms of tinnitus generation may affect auditory temporal resolution in tinnitus patients even with normal audiometry. Thus, studying the correlation between tinnitus characteristics and auditory temporal resolution in subjects with tinnitus may help in proper modification of tinnitus management strategy.

Objective This study aims to examine the relationship between the psychoacoustic measures of tinnitus and the auditory temporal resolution in subjects with normal audiometry.

Methods Two normal hearing groups with ages ranging from 20 to 45 years were involved: control group of 15 adults (30 ears) without tinnitus and study group of 15 adults (24 ears) with tinnitus. Subjective scaling of annoyance and sleep disturbance caused by tinnitus, basic audiological evaluation, tinnitus psychoacoustic measures and Gaps in Noise test were performed. Data from both groups were compared using independent sample t-test. Psychoacoustic measures of tinnitus and Gaps in Noise test parameters of the tinnitus group were correlated with Pearson's correlation coefficient.

Keywords

- tinnitus

- subjective

- auditory processing disorder

- auditory pathway disorders

- central Results Significantly higher hearing threshold, higher approximate threshold and lower correct Gaps in Noise scores were observed in tinnitus ears. There was no significant correlation between psychoacoustic measures of tinnitus and Gaps in Noise test parameters of the tinnitus group.

Conclusion Auditory temporal resolution impairment was found in tinnitus patients, which could be attributed to cochlear impairment or altered neural firing within the auditory pathway. It is recommended to include temporal resolution testing in the tinnitus evaluation battery to provide a proper management planning.
\end{abstract}

\section{Introduction}

Tinnitus includes two main categories: subjective and objective. The subjective category represents $80 \%$ of cases. It is a phantom auditory perception: a perception of sound in absence of a matching acoustic or mechanical correlate in the cochlea. ${ }^{1}$ It is only audible to the patient. On the other

received

January 6, 2016

accepted

March 25, 2016

published online

May 5, 2016

$10.1055 / \mathrm{s}-0036-1583526$ ISSN 1809-9777. hand, the objective tinnitus (20\%) is audible to the patient and to the examiner. Many physicians use the term tinnitus to name subjective tinnitus and the term somato-sound to name objective tinnitus. ${ }^{2}$ Tinnitus represents the main symptom of many diseases. It causes various emotional reactions that may affect the quality of life in different manners. ${ }^{3}$ Measurement, quantification, and description of tinnitus depend on the

Copyright $\odot 2017$ by Thieme-Revinter

Publicações Ltda, Rio de Janeiro, Brazil

License terms

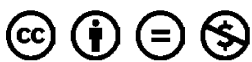


patients' response but it is difficult to be evaluated objectively. Psychoacoustic measures of tinnitus (pitch, loudness) are part of patient symptom characterization. Therefore, they are essential to prescribe some sound-based interventions and to interpret the physiological effect of treatments. ${ }^{4}$

Tinnitus generators are located within the auditory system whether peripherally or centrally. Consequently, different auditory processing abilities, including auditory temporal processing, could be affected. Auditory temporal processing represents the time related aspects of acoustic signal processing. ${ }^{5}$ Precise processing of the sound's temporal aspects is fundamental to the basic processing starting at a neuronal basis to a higher complex stage of speech perception. Specifically, temporal processing skills are crucial to phonemic, lexical, and prosodic distinctions, auditory closure, as well as the ability to understand speech in background noise. ${ }^{6}$ Owing to the basic role of temporal processing in auditory perception, it is possible to partly underling most other auditory processes such as localization, discrimination, pattern recognition, binaural integration, and binaural separation.

Tinnitus interference with auditory perception of speech could be related to defective auditory temporal processing and other auditory processing abilities. ${ }^{7}$ Therefore, evaluating auditory temporal processing in such patients is advisable. Gaps in Noise (GIN) test evaluates the ability of auditory temporal resolution, which is an important skill in speech perception. Auditory temporal resolution is a subcategory of the auditory temporal processing, which refers to the ability of the auditory system to recognize rapid changes in the envelope of sound stimulus overtime. GIN test measures the ability of subjects to detect the minimum intervals of silence in a sound stimulus. Studies have presented the GIN test as an easily administered tool that yields good sensitivity and specificity in adult populations. ${ }^{5,8}$

Based on the notion that the tinnitus could generate either peripherally or centrally, we hypothesized that the auditory processing abilities, including aspects of temporal processing, could be affected, which, in turn, affects speech perception. Therefore, the evaluation process cannot be complete without assessing the effect of tinnitus on the auditory temporal processing mainly the auditory temporal resolution, the most critical aspect of temporal processing. This study is designed to find out such relationship, which can be of help in the modification and future development of remediation programs for temporal processing deficits in tinnitus patients. Moreover, psychoacoustic measures of tinnitus (pitch and loudness) could reflect the severity of pathology underlying tinnitus (whether in the cochlea or in the auditory neural pathway). Auditory temporal processing could be affected by this pathology. This assumption had led us to search for a possible relationship between the psychoacoustic measures of tinnitus and GIN test. Presence of such a relationship would raise the dependence on the more reliable GIN test over the psychoacoustic measures of tinnitus. Otherwise, absence of any relation would reflect different aspects they represent, which requires the inclusion of both tests in tinnitus evaluation.

\section{Methods}

\section{Participants}

The present study included 30 adult individuals (20-45 years) of both genders [17 (56.7\%) females and 13 (43.3\%) males]. They were distributed into two groups: (1) control group consisted of 15 adults ( $30.7 \pm 8.8$ years) with no complaint of tinnitus (a total of 30 ears) and (2) study group consisted of 15 subjects ( $34.7 \pm 7.2$ years; $t=1.355, p=0.186$ ) who had a main complaint of tinnitus. The tinnitus was bilateral in nine subjects, left sided in four subjects, and right sided in two subjects (a total of 24 ears).

Individuals from the control group were volunteers from colleagues and workers while those of the study group were the attendants of Audiology Unit, ENT Department, between October 2015 and January 2016. Participants were informed about the purpose and the structure of the study. All of them provided a signed informed consent. The institutional review board approved the study protocol on October 2015.

All subjects fulfilled the following inclusion criteria:

1. Healthy external ear.

2. Normal middle ear pressure.

3. Normal behavioral hearing thresholds (below or equal to $25 \mathrm{dBHL}$ between 0.25 and $8 \mathrm{kHz}$ ) with normal speech recognition scores (SRS).

Those of the study group expressed:

1. Continuous tinnitus of stationary or progressive course for at least three months.

2. No pulsatile tinnitus by history and clinical examination.

\section{Procedures}

The evaluation started with full history taking involving details about tinnitus side, character, pitch, course, and duration. The next step was subjective scaling of annoyance and sleep disturbance because of tinnitus. The level of annoyance was subjectively reported by tinnitus sufferers on a 5point scale, where $0=$ no annoyance, $1=$ little annoyance, 2 = average annoyance, $3=$ high annoyance, and $4=$ intolerable. Sleep disturbance was rated on a 5-point scale as $0=$ never affected, $1=$ rarely affected, $2=$ sometimes affected, 3 = mostly affected, and 4 = always affected. ${ }^{9,10}$

Clinical and audiological examinations consisted of: 1) audiological evaluation; 2) tinnitus psychoacoustic measures; 3 ) transient evoked otoacoustic emissions (TEOAE); and 4) GIN test.

\section{Audiological evaluation}

Tympanometry, acoustic reflex measurement, pure-tone audiometry (at frequencies from 0.25 to $8 \mathrm{kHz}$ for air conduction and from 0.5 to $4.0 \mathrm{KHz}$ for bone conduction), extended high frequency audiometry (at 10, 12.5, and 16 $\mathrm{kHz}$ ), and speech audiometry [speech reception threshold (SRT), speech recognition scores (SRS) and loudness discomfort level (LDL)]. LDL judgments based on an ascending presentation method. LDL of $95 \mathrm{~dB}$ or greater at all frequencies are considered negative for hyperacusis. LDLs are positive for hyperacusis at $90 \mathrm{~dB}$ or less. 


\section{Psychoacoustic measures of tinnitus}

Measures of tinnitus include tinnitus pitch and loudness matching. ${ }^{3,11}$ Tinnitus matching was performed in the nontinnitus ear when tinnitus was unilateral and in the better ear when tinnitus was bilateral. ${ }^{12}$

\section{Pitch Matching}

Pitch matching is an attempt to quantify tinnitus by its approximate frequency. A stimulus of $1000 \mathrm{~Hz}$ at 5-10dBSL was presented then followed by moving up or down in octave steps, based on patients' report of tinnitus pitch. Once the closest frequency to tinnitus pitch was established, half octave steps were used for more fine estimation.

\section{Loudness Matching}

Loudness matching is the perceptual equivalent of tinnitus sound intensity, defined in terms of decibel. The procedure started at the frequency determined during pitch matching and at a level just below threshold then the intensity was increased in 1-dB steps until the patient signaled a match. The tinnitus loudness match was expressed in dBSL.

\section{Transient-evoked otoacoustic emissions}

The TEOAE was elicited using click stimulus with intensity of $80 \mathrm{dBpeSPL}$. A signal to noise ratio $\geq 3 \mathrm{~dB}$ in at least 4 of the 5 frequencies $1,1.4,2,2.8$, and $4 \mathrm{kHz}$ with a response reproducibility of at least $70 \%$ is considered as passed response. The overall amplitude of the TEOAE was measured to assess outer hair cell function.

\section{Gaps-In-Noise (GIN) test}

The GIN test includes series of white noise segments presented at $50 \mathrm{dBSL}$, lasting six seconds each. Every segment may contain zero to three silence gaps of ten different durations $[2,3,4,5,6,8,10,12,15,20$ milliseconds (ms)]. GIN test comprises four lists, each composed of several segments presenting each gap six times, and thus each list is composed of 60 gaps. $^{5}$ In this study, lists 1 and 2 of the test were presented, one in each ear in a random order to avoid training effect if testing was started every time in certain ear. Subjects were instructed to push a button every time they heard the silence gaps. They were tested while seated in a sound treated booth and stimuli were presented monaurally to both ears through calibrated TDH-39 headphones. Approximate gap detection threshold and percentage of correct responses were obtained. The shortest silence gap noticed by the subject at least four out of six times (67\%) was considered the approximate gap detection threshold. ${ }^{13}$ The percentage of correct responses was calculated over the total number of gabs: (total number of gaps identified/total number of gaps in the list) $\times 100$.

\section{Equipment}

Two channel audiometer Madsen model Orbiter 922 version 2 (Hauppauge, USA) and immittance meter Madsen model Zodiak 902 (USA) were used for audiological investigations. We used the Otodynamics ILOv6 to measure the TEOAE. GIN test $\mathrm{CD}$ was loaded on $\mathrm{CD}$ player that coupled to the twochannel audiometer.

\section{Statistical Analysis}

We tabulated data collected from participants' right and left ears in raw data tables. We statistically analyzed them using the SPSS software statistical computer package version 21 (SPSS Inc., Chicago, Illinois, USA). Simple descriptive analysis was performed to calculate the mean \pm SD of the test variables. We compared the mean values of GIN test measures in control versus study groups using independent sample $t$-test

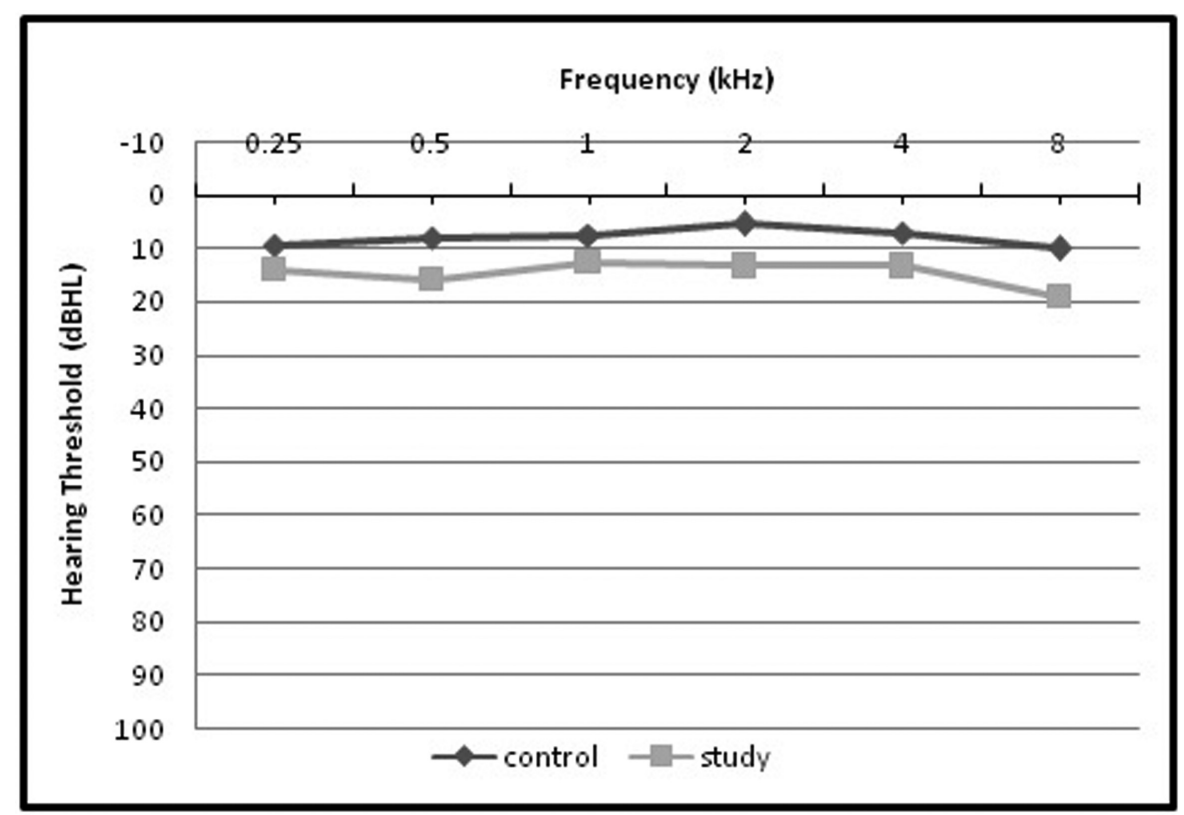

Fig. 1 Mean pure-tone hearing threshold in control versus study groups. 
Table 1 Audiological evaluation (in $\mathrm{dBHL}$ ) and TEOAE (in $\mu \mathrm{V}$ ) in control versus study groups

\begin{tabular}{|c|c|c|c|}
\hline & $\begin{array}{l}\text { Control } \\
(n=30) \\
(\text { mean } \pm S D)\end{array}$ & $\begin{array}{l}\text { Study } \\
(n=24) \\
(\text { mean } \pm S D)\end{array}$ & $t(p)$ \\
\hline $0.25 \mathrm{kHz}$ & $9.5 \pm 2.8$ & $14.2 \pm 4.9$ & $2.432\left(0.029^{*}\right)$ \\
\hline $0.5 \mathrm{kHz}$ & $8 \pm 4.2$ & $15.8 \pm 5.5$ & $3.12\left(0.008^{* *}\right)$ \\
\hline $1 \mathrm{kHz}$ & $7.5 \pm 3.5$ & $12.5 \pm 2.7$ & $2.958\left(0.010^{*}\right)$ \\
\hline $2 \mathrm{kHz}$ & $5.5 \pm 4.9$ & $13.3 \pm 6$ & $2.817\left(0.014^{*}\right)$ \\
\hline $4 \mathrm{kHz}$ & $7 \pm 4.8$ & $13.3 \pm 6.1$ & $2.314\left(0.036^{*}\right)$ \\
\hline $8 \mathrm{kHz}$ & $10 \pm 3.3$ & $19.2 \pm 4.9$ & $4.469\left(0.001^{* *}\right)$ \\
\hline PTA & $7.9 \pm 1.7$ & $14.7 \pm 2.5$ & $5.583\left(0.000^{* * *}\right)$ \\
\hline Extended HFA & $15.7 \pm 3.7$ & $23.8 \pm 1.6$ & $4.05\left(0.003^{* *}\right)$ \\
\hline SRT & $6.5 \pm 2.4$ & $14 \pm 4.2$ & $3.98\left(0.003^{* *}\right)$ \\
\hline TEOAE & $14.11 \pm 2.12$ & $9.76 \pm 5.11$ & $2.16\left(0.05^{*}\right)$ \\
\hline
\end{tabular}

Abbreviations: HFA, high frequency threshold average; PTA, pure-tone threshold average; SD, standard deviations; SRT, speech reception threshold; $t$ $(p)$, t-value and its probability; TEOAE, transient-evoked otoacoustic emissions.

${ }^{*}$ Significant difference when $p=0.05$ to $>0.01$, ${ }^{* *}$ highly significant difference when $p=0.01$ to $>0.001$ and ${ }^{* * *}$ extremely significant difference when $p \leq 0.001$.

to calculate the $t$-value and its $p$. We used Pearson's correlation coefficient to test the presence of correlation between tinnitus psychoacoustic measures and GIN test results. For all tests, statistical significance was set at $p$ value $\leq 0.05$.

\section{Results}

The duration of tinnitus in the study group ranged from 6-36 months with mean of $14.8 \pm 8.1$ months. The median value on subjective scaling of annoyance was 2 (average annoyance), ranging from 1 (little annoyance) to 3 (high annoyance). Whereas subjective scaling of sleep disturbance revealed a median value of 1 (rarely affected) with a minimum of 0 (never affected) and a maximum of 3 (mostly affected).

Significantly higher values were obtained from tinnitus ears as compared with ears of control group in mean pure tone hearing threshold at all frequencies $(0.25$ through $8 \mathrm{kHz})$ ( - Fig. 1), mean pure tone threshold average, mean extended high frequencies threshold average at $10,12.5$, and $16 \mathrm{kHz}$ and SRT ( - Table 1 ). The SRS was $100 \%$ in all subjects. The LDL was $\geq 95 \mathrm{~dB}$ in $18(75 \%)$ tinnitus ears and $95 \mathrm{~dB}$ in the remaining 6 (25\%) ears. Psychoacoustic measures of tinnitus revealed: pitch match ranging from $1-8 \mathrm{kHz}$ with a median of $4 \mathrm{kHz}$ and a mean of $4.78 \pm 2.39 \mathrm{kHz}$ and loudness match ranging from 5-35 dBSL with a median of $15 \mathrm{dBSL}$ and a mean of $18.2 \pm 11.1 \mathrm{dBSL}$. Additionally, overall TEOAE amplitude was significantly higher in the control versus study group (-Table 1).

Comparing GIN test measures in right versus left ears of the control group revealed non-significant differences (-Table 2). Consequently, all ears in the control group were managed statistically as one group. Significant higher approximate GIN threshold and lower percentage of correct responses were found in the study group when compared with the control one ( - Table 3 ).

As shown in - Table 4 , there was no significant correlation between tinnitus measures (pitch and loudness) and various variables including (duration of tinnitus, subjective scaling and audiological profile) except a significant positive correlation between tinnitus pitch and overall TEOAE amplitude. Additionally, no significant correlation was found between GIN test measures in study group and different values including (duration of tinnitus, subjective scaling, audiological profile, and tinnitus psychoacoustic measures) except a significant positive correlation between overall TEOAE amplitude and GIN scores (- Table 5).

\section{Discussion}

Tinnitus is a perception of sound in spite of the absence of external auditory stimulation. ${ }^{1}$ The reported prevalence of chronic tinnitus is $\sim 15 \%$ of the general population. In $\sim 5 \%$,

Table 2 Comparison of GIN test measures in right versus left ears of control group

\begin{tabular}{|l|l|l|l|}
\hline & $\begin{array}{l}\text { Right ears }(\boldsymbol{n}=15) \\
(\text { mean } \pm \text { SD) }\end{array}$ & $\begin{array}{l}\text { Left ears }(\boldsymbol{n}=15) \\
(\text { mean } \pm \text { SD) }\end{array}$ & $p$ \\
\hline Approximate GIN threshold (ms) & $5.4 \pm 0.7$ & $5 \pm 0.9$ & 0.201 \\
\hline Correct score (\%) & $66.2 \pm 4.6$ & $69.01 \pm 4.5$ & 0.102 \\
\hline
\end{tabular}

Abbreviations: GIN, gaps in noise; SD, standard deviations; p, probability. 
Table 3 Comparison of GIN test measures in control versus study groups

\begin{tabular}{|l|l|l|l|}
\hline & $\begin{array}{l}\text { Control group } \\
(\boldsymbol{n}=30 \text { ears) } \\
\text { (mean } \pm \text { SD) }\end{array}$ & $\begin{array}{l}\text { Study group } \\
(\boldsymbol{n}=24 \text { ears) } \\
\text { (mean } \pm \text { SD) }\end{array}$ & $p$ \\
\hline Approximate GIN threshold (ms) & $5.2 \pm 0.8$ & $7 \pm 0.1 .1$ & $0.000^{* * *}$ \\
\hline Correct score (\%) & $67.6 \pm 4.7$ & $58.1 \pm 5$ & $0.000^{* * *}$ \\
\hline
\end{tabular}

Abbreviations: GIN, gaps in noise; $p$, probability; SD, standard deviations.

${ }^{* * *}$ extremely significant difference when $p \leq 0.001$.

Table 4 Correlation between psychoacoustic measures of tinnitus and duration of tinnitus, subjective scaling and audiological profile

\begin{tabular}{|l|l|l|l|l|}
\hline & \multicolumn{2}{|l|}{ pitch } & \multicolumn{2}{l|}{ loudness } \\
\cline { 2 - 5 } & $\boldsymbol{r}$ & $\boldsymbol{p}$ & $\boldsymbol{r}$ & $\boldsymbol{p}$ \\
\hline Duration & 0.779 & 0.068 & -0.450 & 0.371 \\
\hline Annoyance level & -0.454 & 0.366 & 0.415 & 0.413 \\
\hline Sleep disturbance & -0.055 & 0.917 & 0.066 & 0.902 \\
\hline PTA & 0.602 & 0.206 & 0.030 & 0.955 \\
\hline Extended HFA & 0.636 & 0.364 & 0.565 & 0.321 \\
\hline TEOAE & 0.884 & $0.047^{*}$ & 0.226 & 0.715 \\
\hline
\end{tabular}

Abbreviations: HFA, high frequency threshold average; $p$, probability; PTA, pure-tone threshold average; TEOAE, transient-evoked otoacoustic emissions; $r$, correlation value.

*Significant difference when $p=0.05$ to $>0.01$.

severe impact on the quality of life arises including sleep disturbances, speech perception difficulties, anxiety, and depression. ${ }^{14,15}$ Despite the high prevalence of tinnitus, the underlying pathophysiological mechanism remains unknown. Tinnitus generators are theoretically located in the auditory pathway. One of the auditory difficulties experienced in tinnitus is the impaired speech perception due to an underlying auditory temporal processing deficit.

The main findings of the present study were the differences between the tinnitus and control groups in hearing thresholds and GIN test results. Hearing thresholds were higher at all frequencies including the extended high frequencies in the tinnitus group in spite of being within normal limits. Similar results were found in literature. ${ }^{8,16}$ It is unclear whether this finding was because of an underlying subclinical pathology that affects hearing thresholds in tinnitus ears or because of the presence of tinnitus itself. Loss of cochlear amplification occurs when there is a damage of at least $33 \%$ of outer hair cells. ${ }^{17}$ Consequently, we can relate the elevation of hearing threshold in the study group to a subtle cochlear

Table 5 Correlation between GIN test measures and duration of tinnitus, subjective scaling, audiological profile, and tinnitus psychoacoustic measures

\begin{tabular}{|l|l|l|l|l|}
\hline & Approximate GIN threshold & GIN correct scores \\
\cline { 2 - 5 } & $\boldsymbol{r}$ & $\boldsymbol{p}$ & $\boldsymbol{r}$ & $\boldsymbol{p}$ \\
\hline Duration of tinnitus & 0.705 & 0.117 & -0.778 & 0.068 \\
\hline Annoyance level & 0.791 & 0.061 & -0.608 & 0.200 \\
\hline Sleep disturbance & 0.500 & 0.313 & -0.085 & 0.874 \\
\hline PTA & 0.057 & 0.914 & -0.751 & 0.085 \\
\hline Extended HFA & 0.302 & 0.698 & -0.109 & 0.891 \\
\hline TEOAE & -0.387 & 0.520 & 0.910 & $0.032^{*}$ \\
\hline Tinnitus pitch & 0.053 & 0.893 & -0.470 & 0.202 \\
\hline Tinnitus loudness & 0.219 & 0.571 & -0.277 & 0.471 \\
\hline
\end{tabular}

Abbreviations: GIN, gaps in noise; HFA, high frequency threshold average; $p$, probability; PTA, pure-tone threshold average; $r$, correlation value; TEOAE, transient-evoked otoacoustic emissions.

*Significant difference when $p=0.05$ to $>0.01$. 
impairment that is not reflected by the audiogram. Many researchers speculated that the reduced neural output from the impaired cochlea leads to compensatory increase in neural gain in the auditory brainstem (including cochlear nucleus and inferior colliculus) and auditory cortex. ${ }^{18-21}$ This gain could give rise to tinnitus due to amplification of the spontaneous activity of auditory neurons. On the other hand, cochlear impairment could lead to differentiation of central auditory cortex. This can lead to alterations in central plasticity resulting from changing the balance of excitatory and inhibitory reaction of the nerve, which, in turn, generates tinnitus. ${ }^{11}$ Furthermore, the presence of tinnitus could confuse the patients during the process of threshold detection.

This work assessed GIN, a cornerstone process of temporal resolution, in tinnitus patients. Individuals have to make good GIN detection to perform the other two aspects of temporal resolution, which are the modulation detection and the frequency discrimination. Regarding performance on GIN test, there were an elevation of approximate GIN thresholds and the reduction of GIN correct scores in tinnitus group as compared with normal subjects. These findings are in consistency with results of other studies where GIN test performance was impaired in normal hearing subjects with tinnitus. $^{7,8,22}$ We anticipated that the impaired temporal resolution could be related to tinnitus generating insult within the auditory system, either cochlear, central, or both. The proper temporal resolution of complex auditory signals such as speech starts with the silence gaps coding, spectral coding and modulation detection within the cochlea and then conserved in the ascending auditory pathway. The silence gaps are coded in the cochlea as time intervals between action potentials at the base of inner hair cells. The presence of minimum cochlear insult underlying tinnitus, even with normal pure tone audiometry, may result in prolongation of time intervals required for coding of acoustic signals. Thus, the precise coding of the signal is disrupted.

Additional explanation can be afforded on a basis of temporal desynchronization and auditory nerve deafferentation. ${ }^{23,24}$ Each afferent nerve fiber within the auditory nerve responds stochastically to the sound (the inner hair cell receptor potential). Thus, the aggregated neural response represents a neural coding of the stimulus waveform. The presence of cochlear insult disrupts the neural waveform encoding by means of temporal desynchronization and/or differentiation with subsequent deterioration of auditory perception. Mainly, desynchronization impairs speech intelligibility in noise ${ }^{23}$ whereas differentiation decreases the velocity of stimulus coding as well as degrades the neural encoding of low intensity and high frequency waveform features. $^{24}$

Cochlear affection is supported by the significant elevation of hearing threshold at all frequencies including the extended high frequencies, and the significant reduction of TEOAE amplitude in tinnitus patients, as evidenced in the current study and in literature. ${ }^{8,16,25}$ Otoacoustic emissions originate from properly functioning outer hair cells. Thus, the presence of cochlear dysfunction will cause reduction or even absence of the otoacoustic emissions. A subclinical cochlear pathology affecting up to $33 \%$ of the outer hair cells could reduce the otoacoustic emissions in spite of absence of any audiometric evidence. ${ }^{17,26}$ Our results revealed that ears with more enhanced emissions had better GIN scores. Theoretically, the enhanced amplitude of TEOAE would be an indicator of cochlear integrity with better signal encoding.

In addition, it was reported that altered afferent input and/ or deficits in neural structures might result in tinnitus perception. ${ }^{27}$ This higher order dysfunction in the neural correlates of tinnitus could be a reason for impaired temporal resolution. Even the chronic exposure to tinnitus as an internal phantom sound may cause modulations or alterations within the auditory pathway that could adversely affect the temporal resolution.

Data obtained from this study revealed that psychoacoustic measures of tinnitus were not in correlation with the subjective scaling that represents patient's reaction to tinnitus. This suggests that the subjective impact of tinnitus is not dependent on matched pitch or loudness. Moreover, we found no correlation between GIN test parameters and psychoacoustic measures of tinnitus. We speculated that the absence of correlation between tinnitus measurements and both of the subjective impact of tinnitus and GIN test parameters weakened their role as markers in sound therapy of tinnitus. Thus, the concept of management planning that depends on tinnitus measurements alone may not improve the patient's reaction to tinnitus or the auditory temporal resolution. Adding a test for temporal resolution such as GIN test to the tinnitus evaluation battery that includes tinnitus measurements and assessment of subjective impact of tinnitus will be more beneficial as a guide for adding a suitable remediation therapy to the tinnitus management protocol to improve speech perception in subjects with tinnitus.

\section{Conclusion}

The GIN detection process was impaired in tinnitus patient, which consequently could affect modulation detection and frequency discrimination process, all of which will result in poor temporal resolution. Temporal resolution impairment was not in correlation with the psychoacoustic measures or the subjective perception of tinnitus. This requires an evaluation of temporal resolution as a complementary for tinnitus assessment battery. These findings could be of clinical importance to select a suitable protocol of tinnitus management in normal hearing individuals.

\section{References}

1 Jastreboff PJ. Phantom auditory perception (tinnitus): mechanisms of generation and perception. Neurosci Res 1990;8(4): 221-254

2 Dobie RA. Overview: suffering from tinnitus. In: snow JB, ed. Tinnitus: theory and management. Ontario: Decker BC, Inc; 2004: $1-7$

3 Henry JA, Zaugg TL, Schechter MA. Clinical guide for audiologic tinnitus management I: Assessment. Am J Audiol 2005;14(1): $21-48$ 
4 Hoare DJ, Edmondson-Jones M, Gander PE, Hall DA. Agreement and reliability of tinnitus loudness matching and pitch likeness rating. PLoS ONE 2014;9(12):e114553

5 Musiek FE, Shinn JB, Jirsa R, Bamiou DE, Baran JA, Zaida E. GIN (Gaps-In-Noise) test performance in subjects with confirmed central auditory nervous system involvement. Ear Hear 2005; 26(6):608-618

6 Reed CM, Braida LD, Zurek PM. Review article: review of the literature on temporal resolution in listeners with cochlear hearing impairment: a critical assessment of the role of suprathreshold deficits. Trends Amplif 2009;13(1):4-43

7 Mehdizade Gilani V, Ruzbahani M, Mahdi P, et al. Temporal processing evaluation in tinnitus patients: results on analysis of gap in noise and duration pattern test. Iran J Otorhinolaryngol 2013;25(73):221-226

8 Sanches SGG, Samelli AG, Nishiyama AK, Sanchez TG, Carvallo RMM. GIN Test (Gaps-in-Noise) in normal listeners with and without tinnitus. Pro Fono 2010;22(3):257-262

9 Andersson G, McKenna L. The role of cognition in tinnitus. Acta Otolaryngol Suppl 2006;126(556):39-43

10 Gudwani S, Munjal SK, Panda NK, Verma RK. Correlation of tinnitus loudness and onset duration with audiological profile indicating variation in prognosis. ISRN Otolaryngol 2013;2013:205714

11 Powers L, Ramirez T. Clinical management of tinnitus [Internet]. Audiol Online 2014; Article ID 12558. Available at: http://www. audiologyonline.com

12 De Ridder D, Congedo M, Vanneste S. The neural correlates of subjectively perceived and passively matched loudness perception in auditory phantom perception. Brain Behav 2015;5(5):e00331

13 Shinn JB, Chermak GD, Musiek FE. GIN (Gaps-In-Noise) performance in the pediatric population. J Am Acad Audiol 2009;20(4):229-238

14 Kochkin S, Tyler R, Born J. MarkeTrak VIII: The prevalence of tinnitus in the United States and the self-reported efficacy of various treatments. Hear Rev 2011:10-26. Available at: www. hearingreview.com.

$15 \mathrm{Kim} \mathrm{H-J,} \mathrm{Lee} \mathrm{H-J,} \mathrm{An} \mathrm{S-Y,} \mathrm{et} \mathrm{al.} \mathrm{Analysis} \mathrm{of} \mathrm{the} \mathrm{prevalence} \mathrm{and}$ associated risk factors of tinnitus in adults. PLoS ONE 2015;10(5): e0127578
16 An Y-H, Jin SY, Yoon SW, Shim HJ. The effects of unilateral tinnitus on auditory temporal resolution: gaps-in-noise performance. Korean J Audiol 2014;18(3):119-125

17 Chen GD, Tanaka C, Henderson D. Relation between outer hair cell loss and hearing loss in rats exposed to styrene. Hear Res 2008; 243(1-2):28-34

$18 \mathrm{Gu}$ JW, Herrmann BS, Levine RA, Melcher JR. Brainstem auditory evoked potentials suggest a role for the ventral cochlear nucleus in tinnitus. J Assoc Res Otolaryngol 2012;13(6): 819-833

19 Schaette R, McAlpine D. Tinnitus with a normal audiogram: physiological evidence for hidden hearing loss and computational model. J Neurosci 2011;31(38):13452-13457

20 Hickox AE, Liberman MC. Is noise-induced cochlear neuropathy key to the generation of hyperacusis or tinnitus? J Neurophysiol 2014;111(3):552-564

21 Plack CJ, Barker D, Prendergast G. Perceptual consequences of "hidden" hearing loss. Trends Hear 2014;18:1-11

22 Sanches SG, Sanchez TG, Carvallo RM. Influence of cochlear function on auditory temporal resolution in tinnitus patients. Audiol Neurootol 2010;15(5):273-281

23 Pichora-Fuller MK, Schneider BA, Macdonald E, Pass HE, Brown S. Temporal jitter disrupts speech intelligibility: a simulation of auditory aging. Hear Res 2007;223(1-2):114-121

24 Lopez-Poveda EA, Barrios P. Perception of stochastically undersampled sound waveforms: a model of auditory deafferentation. Front Neurosci 2013;7(124):124

25 Emadi M, Rezaei M, Najafi S, Faramarzi A, Farahani F. Comparison of the transient evoked otoacoustic emissions (TEOAEs) and distortion products otoacoustic emissions (DPOAEs) in normal hearing subjects with and without tinnitus. Indian J Otolaryngol Head Neck Surg 2015; doi: 10.1007/s12070-015-0824-9

26 Mokrian H, Shaibanizadeh A, Farahani S, et al. Evaluation of distortion and transient evoked otoacoustic emission in tinnitus patients with normal hearing. Iran J Otorhinolaryngol 2014; 26(74):19-24

27 Bartels H, Staal MJ, Albers FWJ. Tinnitus and neural plasticity of the brain. Otol Neurotol 2007;28(2):178-184 